\title{
Ekelmanagement. Professioneller Umgang mit Ekel in der Kita
}

\author{
Dorothee Gutknecht
}

Evangelische Hochschule Freiburg

\section{Einleitung}

In der Früh- und Elementarpädagogik haben die Pflegeaufgaben in den letzten Jahren deutlich zugenommen. Die wachsende Betreuung von Kindern unter drei Jahren, die Ganztagsbetreuung aber auch der Anspruch Inklusion sind hier wesentliche Ursachen. In der Betreuung von jungen Kindern, aber auch von Kindern mit Erkrankungen, Behinderungen oder Einschränkungen ist ein höherer pflegerischer Assistenzbedarf die Regel. Die Kindertageseinrichtungen sind hierauf meist noch ungenügend eingestellt. Es fehlt oft schon am Equipment: So gibt es zum Beispiel in der Regel weder Stehwickeltische, die ein entwicklungsgerechtes Wickeln ermöglichen, noch eine angemessene ergonomische Ausstattung der Waschräume. Hydraulische Wickeltische, die sich an die Körpergröße der Pädagogin anpassen lassen, sind die große Ausnahme auch dann, wenn Kinder im Altersspektrum bis sechs Jahre pflegerisch versorgt werden müssen (Gutknecht et al., 2018).

Pädagogische Fachkräfte sind zudem in der Regel weder durch ihre Ausbildung noch durch passgenaue Weiterbildungen auf die vermehrten Pflegetätigkeiten vorbereitet. Jahrzehntelang konnten beispielsweise Kinder, die "noch nicht sauber waren“, mit eben dieser Begründung vom Kindergartenbesuch ausgeschlossen werden. Eine solche Praxis verträgt sich nicht mit dem heutigen Anspruch der Inklusion. Auch die Erzieher-Kind-Relation ist vielfach noch nicht darauf ausgelegt, dass ein hoher Pflegeaufwand durch den Assistenzbedarf mehrerer Kinder entstehen kann. Dies betrifft nicht nur die Wickel- oder Toilettensituationen, sondern auch die Assistenz in Lebensaktivitäten wie Essen, Schlafen, An- und Ausziehen. Eine professionelle Auseinandersetzung mit den so genannten Care-Aufgaben ist gerade im Kontext Inklusion zwingend erforderlich.

\section{Ekel in Pflegeprozessen}

Manche Problemlagen, die bisher nur in den Pflegewissenschaften diskutiert worden sind, müssen durch die Zunahme an Pflegetätigkeiten in Kinder- 
tageseinrichtungen auch im frühpädagogischen Bereich reflektiert werden. Hier ist insbesondere der professionelle Umgang mit Ekel-Situationen zu nennen. Auch pädagogische Fachkräfte machen unvermeidlich Erfahrungen mit der Emotion Ekel im Alltag. Sie sind zuständig für Kinder, die gewickelt werden müssen, die Assistenz bei den Toilettensituationen benötigen, vom Po abputzen bis zum Händewaschen. Sie erleben Kinder, die pupsen, rülpsen und verschleimt husten, die Nasensekret absondern, die Hilfe beim Nase putzen benötigen und die übelriechenden Speichelfluss, eitrige Wunden oder auch Mundgeruch haben können. Kotschmieren ist ein Phänomen, das nicht nur bei Kindern mit psychischen Erkrankungen auffallen kann.

Die Auseinandersetzung mit der Emotion Ekel ist deshalb so elementar wichtig, da internationale Forschungsergebnisse zeigen, dass insbesondere Kinder, die noch keine Ausscheidungsautonomie erreicht haben, in Institutionen ein deutlich erhöhtes Risiko haben, Opfer von Übergriffen durch pädagogische Fachkräfte zu werden (vgl. Ringel, 2017; Gutknecht, 2020 unter Verweis auf Yoo, 2012, Gutknecht \& Haug-Schnabel, 2019).

Es besteht zudem das Risiko für die pädagogischen Fachkräfte selbst von hoch belastenden Pflegesituationen mit Ekelepisoden betroffen zu sein und in diesen Situationen mangels Schulung unprofessionell zu handeln. Pflegewissenschaftler*innen haben in den vergangenen beiden Jahrzehnten die Gefahren einer Verdrängung der Emotion Ekel professionstheoretisch herausgearbeitet: Verleugneter Ekel kann sowohl zur Gewalt gegen Patient*innen führen als auch zum Burnout bei den Pflegefachkräften (Ringel, 2017; Pernlochner- Kügler, 2004, Jettenberger, 2017).

In den Pflegewissenschaft gilt das Thema Ekel in der Pflege als unterforscht und immer noch als Tabu. Doch auch im Früh- und Elementarbereich handelt es sich bei Fragen zum Ekel um ein Tabuthema,

- zu dem nur in ersten Anfängen ein Fachdiskurs im Feld über einschlägige Fachzeitschriften besteht (Gutknecht, 2020),

- zu dem es kaum nennenswerte kindheitspädagogische Forschung gibt.

\section{Ekel und seine Funktionen}

In der Psychologie gibt es eine etablierte Ekel-Forschung als Teil der Emotionsforschung. Ekel wird hier den sogenannten Primäraffekten oder Basisemotionen zugeordnet (Izard, 1999, Mertens, 2014, Holodynski, 2006). Der Ekelausdruck tritt dabei universell auf und kann somit auch kulturübergreifend verstanden werden (Ekman, 2010). Izard (1999) beschreibt Ekel als Widerwillen, der mit körperlicher Übelkeit und schlechtem Geschmack im Mund einhergehen kann. Als typisch wird von ihm benannt, dass der vom Ekel-Affekt betroffene Mensch das Bedürfnis hat, sich vom Ekelobjekt möglichst rasch zu entfernen, sich abzuwenden oder wegzudrehen.

Ekel hat grundsätzlich eine außerordentlich wichtige Schutzfunktion, denn er warnt vor Gefahren. So können der Geruch (zum Beispiel faulig), 
der Geschmack (insbesondere bitter) und die Konsistenz (zum Beispiel gallertartig) von verdorbenen Lebensmitteln Ekel auslösen, was mit starken körperlichen Empfindungen des Abscheus verbunden ist. Es bildet sich vermehrt Speichel, was sich zu intensiver Übelkeit bis hin zum Erbrechen steigern kann. Es kommt zu automatischen Abwehrreaktionen. Kalter Schweiß kann ausbrechen, Kurzatmigkeit, Blutdruckerhöhung und Herzklopfen können auftreten (Pernlochner-Kügler, 2004).

Das typische, so genannte Ekelgesicht, mit der Hebung der Oberlippe wird kulturübergreifend in Ekel-Situationen gezeigt und hat eine Signalwirkung auf die Beteiligten in der Umgebung. Es löst nicht zuletzt durch die Spiegelneuronen und die menschliche Fähigkeit zur Empathie (Häusser, 2012; Rizzolatti et al., 2008) ebenfalls Abwehr und das Bedürfnis nach Distanzierung von der Ekel auslösenden Substanz aus. Der Ekel-Affekt zeigt von der frühesten Kindheit an, was toxisch, infektiös und ungenießbar, also zu meiden ist. Ausgeprägter und starker Ekel ist dabei kaum zu unterdrücken, denn der hier oft ausgelöste Brechreiz wird vom vegetativen Nervensystem gesteuert und ist somit kaum über kognitive Strategien beeinflussbar. Anders ist dies bei kulturell erworbenem Ekel, zum Beispiel vor essbaren Insekten als Nahrungsquelle, dies kann durchaus über kognitive Prozesse des Verlernens und Umlernens überwunden werden. Ekel ist somit einerseits eine angeborene Basisemotion, andererseits aber auch das Ergebnis von Sozialisation, über die bestimmte Objekte zum Ekelobjekt werden. Ekel vor bestimmten Substanzen wird auch in Interaktionen gelernt. Erwachsene zeigen dem Kind ihr Ekelgesicht und signalisieren ihm auf diese Weise: „Diese Substanz ist gefährlich.“

\section{Ekel in der Entwicklung}

Manche Kinder führen in der Kita Handlungen aus, die von den pädagogischen Fachkräften als überaus eklig empfunden werden. So zeigen sie vielfach keinerlei Ekel vor ihren Körperprodukten. Sie beugen sich tief über die Toilettenöffnung und betrachten ihren Kot, weinen sogar, wenn er weggespült wird. Andere berühren ihre Ausscheidungen, nehmen sie in die Hand, schmieren damit herum. Ekligen Geruch scheinen sie gar nicht zu bemerken. Sie nehmen die Ausscheidungen vielmehr als Teil ihres Körpers wahr, vor dem es keinerlei Grund gibt sich zu ekeln (Pernlochner-Kügler, 2004). Dennoch kann aus diesem Verhalten nicht rückgeschlossen werden, dass Ekel ein anerzogenes Gefühl ist. Pernlochner-Kügler (2004) betont hier in Abgrenzung zu vielen Autor*innen aus dem psychoanalytischen Kontext, dass Kinder, wenn sie beispielsweise Kot in den Mund nehmen, nach dieser unangenehmen Erfahrung, dieses Verhalten nicht wiederholen, somit also keineswegs nur anerzogen handeln.

Ekel kann über alle Sinneskanäle ausgelöst werden über eklige Gerüche, ekligen Geschmack, eklige haptische Erfahrungen sowie über ekelhafte Sehund Höreindrücke. Seh- und Höreindrücke, die Ekel auslösen, sind dabei meist mit Erfahrungen und kognitiven Einsichten verbunden. Hierzu zählt 
der Anblick von Schimmel oder das Hören der Körpergeräusche von anderen Personen beim Urinieren oder Defäkieren auf einer öffentlichen Toilette. In vielen asiatischen Ländern wird über High-Tech-Toiletten Scham und Ekel reduziert, denn hier kann auf Knopfdruck z.B. Wasserfallrauschen eingestellt werden und so die Toilettengeräusche übertönen. Der Hauptsinn für die Ekel-Emotion ist der Geruchssinn. Er zählt zudem zu den ältesten Sinnen, dessen Eindrücke tief im Gedächtnis abgespeichert werden. Riechen und schmecken sind eng verbundene Sinneseindrücke, die zu sofortigen und heftigen Ekelreaktionen wie zum Beispiel Erbrechen führen können. Hier wird die Warn- und Schutzfunktion ganz deutlich.

\section{Der Zusammenhang von Ekel und Scham}

Anders als die Basisemotion Ekel gehört die Emotion der Scham zu den so genannten sozialen Emotionen (Holodynski, 2006). Diese Emotionen entstehen im kognitiven Entwicklungsprozess, in dem ein Kind zunehmend realisiert, welche Verhaltensweisen seinen Bezugspersonen gefallen oder nicht. Schon mit circa zwei Jahren (ebd. 2006) kann ein Kind intensive Scham empfinden, wenn es von seinen Bezugspersonen verlacht, ausgeschimpft oder ausgeschlossen wird, weil es eingenässt oder eingekotet hat. Die Scham ist umso stärker, je größer die Befürchtung ist, die Liebe der wichtigen Bezugspersonen zu verlieren oder mit einer Strafe rechnen zu müssen (Gutknecht \& Haug-Schnabel, 2019). Sobald Anzeichen beginnender Scham beim Kind zu beobachten sind, müssen sie als Entwicklungssignal verstanden werden und konsequent eine Verhaltensänderung bei den Erwachsenen nach sich ziehen. Nach Gutknecht und Haug-Schnabel (2019, S. 52ff) hat die Fähigkeit Sich-schämen zu können einen positiven Sinn: Das Kind spürt hier, eine für sich selbst akzeptable Balance zwischen Nähe und Distanz finden zu können.

\section{Ekel-Emotionen als Auslöser von Aggression und Gewalt}

Internationale Forschungsergebnisse zeigen, dass ältere Kinder, die noch nicht ausscheidungsautonom sind, in Institutionen ein hohes Misshandlungsrisiko haben (Gutknecht, 2020 unter Bezug auf Yoo, 2012).

Fachkräfte können gerade beim Einkoten von Kindern teilweise vollkommen außer sich vor Ekel geraten und jedes Maß in ihrer Reaktion verlieren. Das betroffene Kind hat das Risiko in einem solchen Moment von der Fachkraft nicht mehr als Subjekt, sondern als ein Ekel erregendes Objekt wahrgenommen zu werden. Das Kind erlebt dadurch die Antwortregister von Wut und Feindseligkeit (zur Theorie der Antwortregister siehe Waldenfels, 2007), was eine verstörende Wirkung haben kann. Bereits Izard zeigte die Trias von Ekel, Zorn und Verachtung auf (1999). In der Abbildung ist das so genannte Feindseligkeitsdreieck mit den Auswirkungen Aggression und Gewalt graphisch dargestellt (Gutknecht, 2021; Izard, 1999). 


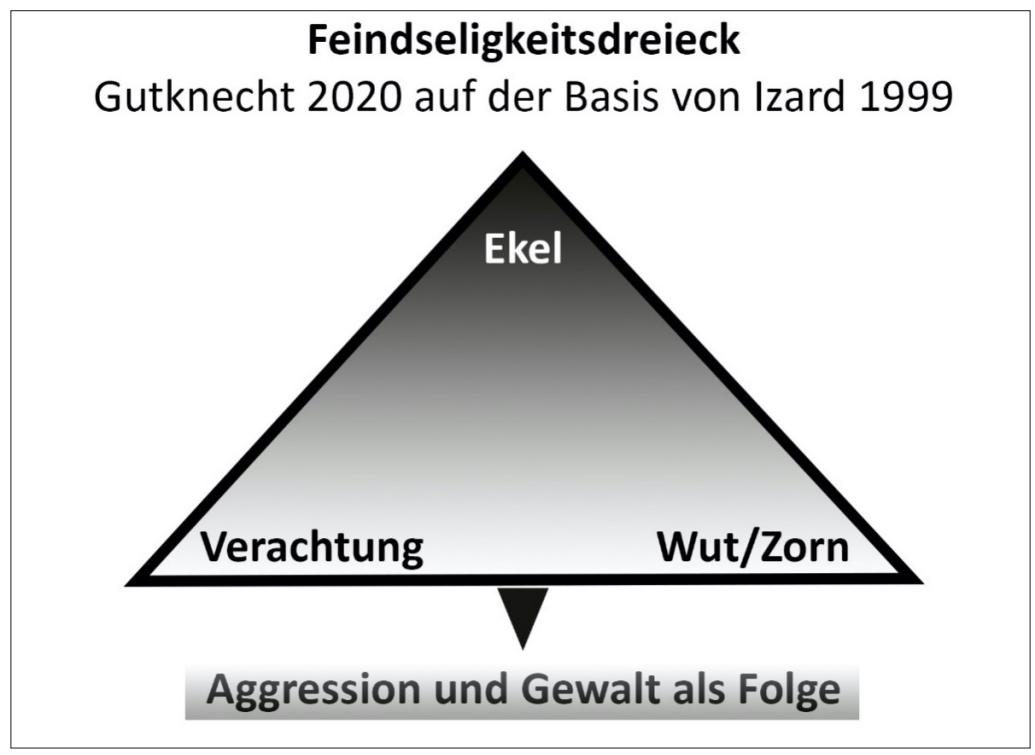

\section{Ekelbotschaften vermeiden}

Über Mimik und Gestik aber auch über Berührungen können pädagogische Fachkräfte in der Kindertageseinrichtung den Kindern Angenommensein und Geborgenheit kommunizieren, aber eben auch Ablehnung und Ekel (Rohrmann \& Wanzeck-Sielert, 2018). Den Fachkräften ist das meist nur allzu bewusst. Es ist oft ein großes Bemühen im Feld zu sehen, dass ein Kind in der Gruppenbetreuung - selbst dann, wenn Ekelgefühle im Spiel sind - auf gar keinen Fall die Botschaft aufnehmen soll: „Ich ekle mich vor dir!". Vor diesem Hintergrund ist auch zu erklären, warum die Verwendung oder Nicht-Verwendung von Handschuhen beim Wickeln von Kleinkindern vielfach wie eine Art „Glaubensfrage“ diskutiert wird. In ihrem Buch Windel ade. Kinder in Krippe und Kita achtsam begleiten (2019) fassen Gutknecht und Haug-Schnabel, ihre Beobachtungen aus Forschungsprojekten wie folgt zusammen:

„Hier kommt es sehr darauf an, wie die Fachkraft das Kind durch die Situation begleitet, denn auch mit Handschuhen lässt sich ein spielerischer, zugewandter Interaktionsstil umsetzen. Fachkräfte, die ohne Handschuhe wickeln möchten, sollten grobe Verschmutzungen mit einem Einmalhandtuch entfernen, die Hände waschen und desinfizieren. Der Verzicht auf Handschuhe ist kein Garant für eine partizipative und responsive Begegnung in der Wickelsituation." (ebd., 2019, S. 71) 


\section{Hohe Ekelsensitivität von Fachkräften kann Fürsorgeverhalten reduzieren}

Wie stark Sinnesreize wie üble Gerüche, unangenehme Haptik, widerwärtiger Geschmack wahrgenommen werden unterscheidet sich von Mensch zu Mensch und hängt auch an der individuellen Sensitivität und Art der Wahrnehmungsverarbeitung.

In einer pflegewissenschaftlichen Studie untersuchten Özkan, Taylan, Adibelli \& Yilmaz (2009) ob sich die Ekelsensitivität auf das Fürsorgeverhalten von angehenden Pflegefachkräften auswirkt. Pflegeschüler*innen haben in der klinischen Praxis häufig Kontakt mit Ekelauslösern, wie z.B. Fäkalien, Schleim oder Urin. Das Forschungsteam vermutete, dass ein hoher Grad an Ekelempfindlichkeit sich direkt auch auf das Pflegeverhalten auswirken könnte. In der Studie wurde ein deskriptives Querschnittsdesign verwendet. Es wurden Daten von 577 Krankenpflegeschüler*innen eines türkischen Universitätskrankenhauses erhoben. Das Erleben der Emotion Ekel wurde von fast $60 \%$ der Teilnehmenden berichtet.

Wichtiges Ergebnis dieser Studie war: Je höher die Ekelempfindlichkeit, desto weniger fürsorgliche Verhaltensweisen zeigten die Pflegefachschüler*innen in ihrer Pflegetätigkeit. Fürsorgliche Verhaltensweisen werden danach durch eine hohe Ekelsensitivität negativ beeinflusst. Das Ergebnis dieser Studie (Ökzan et al., 2009) macht deutlich, wie wichtig es ist, dass bereits in Ausbildung oder Studium die Ekelsensitivität von Fachschüler*innen respektive Studierenden eingeschätzt werden muss. Bereits hier muss an der berufsbezogenen Emotionsregulation gearbeitet werden (Krey, 2015).

In einem Forschungsreview von Hadjittofi, Gleeson \& Arber (2020) zu den Ekel-Erfahrungen professionell Pflegender wurden insbesondere Einfühlung und Empathie als Hilfen zum Umgang mit und Überwindung von Ekel genannt. Dies steht im Widerspruch zu anderen Aussagen im Feld, die Risiken von sozialer Kälte betonen, die sich einstellt, wenn die betroffene Fachkraft am Anspruch „Empathie“ gescheitert ist. Es gilt Ekelemotionen zu erkennen und auch anzusprechen sowie sich mit Maßnahmen eines professionellen Ekelmanagements auseinandersetzen.

\section{Das Erfordernis eines professionellen Ekelmanagements in Kindertageseinrichtungen}

Im Folgenden werden Möglichkeiten beschrieben, die für ein Ekelmanagement explizit in pädagogischen Kontexten für wesentlich erachtet werden. Hier sind Erkenntnisse aus Pflegewissenschaften, Kindheitspädagogik, Psychologie, Medizin und Philosophie handlungsleitend, da bei der Komplexität der Thematik eine interdisziplinäre Perspektive zentral ist. 


\section{Analyse unterschiedlicher Ekelsituationen in der Kindertageseinrichtung}

Im Rahmen von Teamgesprächen sollten alle Ekelsituationen, die sich im institutionellen Alltag ereignen, sorgfältig analysiert und dokumentiert werden. Dabei muss möglichst systematisch vorgegangen werden: Jeder Raum, jeder wichtige Bereich der Kita sollte dabei betrachtet werden. Dies gilt auch für Außenräume und -bereiche. Es ist wichtig zu erfassen, welche Situationsbereiche als problematisch wahrgenommen werden. Analysefragen sind hier in Anlehnung an Pernlochner-Kügler (2004) insbesondere:

- Welche Wahrnehmungsbereiche sind in den identifizierten Situationen bei den einzelnen Teammitgliedern besonders betroffen (Tast-, Geruchs-, Geschmacks-, Seh- und Hörsinn)?

- Wen belastet was wie sehr?

- Wer kann wen entlasten?

- Wo kann zur Entlastung angeknüpft werden?

\section{Analyse der Ausstattung}

Wenn sich die Analyse auf Ausstattungsthemen fokussiert, ist von Relevanz, durch welche Ausstattungsmerkmale potenzielle Ekelsituationen abgemildert werden können. Zentrale Aspekte sind hier insbesondere:

- Nutzung frischer klarer Farben im Raum, da Mischfarben bräunlich, gelblich, grünlich Assoziationen mit ekelerregenden Substanzen begünstigen können (vgl. auch Ringel, 2017, Pernlochner-Kügler, 2004)

- gute Belüftung der Räume, insbesondere der Waschräume und Wickelbereiche,

- leichter und unproblematischer Zugang zu Hilfsmitteln,

- ergonomische Optimierung der Ausstattung, Vorhandensein und Anordnung von genügend großen Waschbecken, Duschen, Abfluss, Toiletten, Urinalen,

- Stehwickeltischen, um entwicklungsgerechtes Wickeln der Kinder im Stehen zu ermöglichen (Gutknecht et al., 2018),

- Personaldusche,

- Vorhandensein von Materialien zum Körperschutz, denn diese können Ekelgefühle deutlich reduzieren helfen. Gemeint sind hier z.B. Handschuhe, Schutzkleidung, Mundschutz oder Papier. Eine offen kommunizierte und selbstverständliche Nutzung ist dabei wichtig.

\section{Spezielles Geruchsmanagement}

Die Belastung durch ekelerregende Gerüche muss als sehr hoch eingestuft werden, auch wenn es hier individuelle Unterschiede in der Empfindlichkeit unter den pädagogischen Fachkräften gibt. In einer Arbeitsgruppe des Frühpädagogik Netzwerks Qualität in Kinderkrippen und in der Kinderta- 
gespflege an der Evangelischen Hochschule Freiburg, berichteten Fachkräfte, dass sie unangenehme Gerüche oft als „anhaftend“ erleben und sie nach Ekel-Erlebnissen auch nach Arbeitsschluss quasi noch „in der Nase haben“. Der Geruch, der allem anzuhaften scheint, muss darum gezielt entfernt werden. Günstig ist das Vorhandensein einer Personaldusche und von Spinden, in denen Fachkräfte Wechselwäsche unterbringen können. Auch Berufskleidung kann eine Lösung sein.

In der Situation selbst kann das Team entscheiden, ob eine Experimentierphase mit Düften gestartet werden soll, wo gezielt Gegengerüche eingesetzt werden zum Beispiel Raumsprays, gut riechende Reinigungsmittel, ätherische Öle. Auch die Verwendung von Pfefferminzöl unter der Nase kann eine Lösung sein. Bei allen sich im Raum ausbreitenden Gerüchen kann es allerdings zu unangenehmen und „schwierigen“ Duftmischungen kommen, die den Ekel bei einzelnen Teammitgliedern noch weiter steigern können. Daher muss dieses Vorgehen immer im Rahmen eines explorativen Teamprozesses erfolgen.

\section{Perspektivwechsel auf die Abläufe und das professionelle Handling}

Tätigkeiten wie die Assistenzhandlungen beim Essen, beim An- und Ausziehen oder auch beim Wickeln erfordern von den Fachpersonen ein achtsames und responsives Handling (Gutknecht et al. 2018). Bei dieser responsiven Assistenz wird Kindern viel Raum zur Kooperation gegeben. Die Bewegungsabläufe sind langsam und ganz auf das Kind abgestimmt. Es hilft Ekelgefühle $\mathrm{zu}$ reduzieren, wenn sich die pädagogische Fachkraft auf ihre Handlungen und auf ihre Aufgabe konzentriert, dem Kind möglichst viele Kooperationsmöglichkeiten einzuräumen. Das Kind soll von ihr gut durch die Situation geführt werden. Dabei liegt die Konzentration ganz auf den Bewegungsabläufen und auf der Herstellung gemeinsam geteilter Aufmerksamkeit. Das in der Pikler-Säuglings- und Kleinkindpädagogik verwendete Konzept der Choreographie der Pflege (Gutknecht, 2021; Kállo, 2015) ist hier ebenfalls außerordentlich hilfreich. Die Konzentration auf die Choreographie kann der pädagogischen Fachkraft dabei helfen, belastende Ekel-Emotionen in dem Moment mit dem Kind stärker auszublenden.

\section{Erhöhung der kindlichen Compliance durch Partizipation und gut gestaltete Mikrotransitionen}

Mangelnde Compliance eines Kindes, das eingekotet hat, kann beim Wickeln zu ekelerregenden Situationen führen. Es gibt deutlich mehr Ekelepisoden, wenn das Kind sich insbesondere gegen das Liegen massiv wehrt. Manche Kinder fühlen sich hier gegen ihren Willen in eine Passivität gezwungen, gegen die sie mit aller Macht ankämpfen. Es ist hier sehr wichtig, 
dass sich die pädagogische Fachkraft nicht in einen Machtkampf verstricken lässt. Möglicherweise kann sie sich zwar durchsetzen, aber das Muster permanenter Wickelsituationen ohne Compliance verfestigt sich. Nicht selten verteilt sich dabei der Kot überall: Er ist in den Haaren des Kindes, auf der Wickelauflage, an der Fachkraft, was den Ekel massiv verschärft.

Durch freundliche Ankündigungen „Gleich wirst du gewickelt!“ kann auch ein Kind mit einer Beeinträchtigung, dass sich kognitiv nur langsam auf Situationsänderungen einstellen kann, lernen, Übergänge zu verstehen. Es fällt ihm dann meist leichter auch mit den Spielunterbrechungen zurechtzukommen, die oft mit den Mikrotransitionen verbunden sind, den kleinen Übergängen im Alltag von einer Situation in die andere (Gutknecht, 2018). Konzentration auf klare, ruhige und freundliche Abläufe vermindert Ekel.

Kindern kommt es entgegen, wenn sie grundsätzlich einbezogen werden und sich im Rahmen ihrer Möglichkeiten an allen Handlungen aktiv beteiligen können. Dies erfordert, dass die wachsenden Fähigkeiten des Kindes berücksichtigt werden. Ein Kind, das bereits stehen kann, hat vielleicht Freude daran auch ein neues Skript zum Wickeln aufzubauen, bei dem das Stehen als Ressource eingebracht werden kann. Sehr geeignet dafür ist die Nutzung eines Stehwickeltisches, wie es in der Pikler-Pädagogik umgesetzt wird (Gutknecht, 2021).

\section{Umdeuten der typischen Ekel-Antwort}

Manche Fachkräfte empfinden Scham darüber, wenn sie die typischen Ekel-Antworten ihres Körpers, wie beispielsweise Abwenden etc., spontan zeigen. Das Schamgefühl verstärkt dann die empfundene Belastung noch. Hier kann es hilfreich sein, wenn dies sicherheitstechnisch möglich ist, das Abwenden als Minimal-Auszeit für sich positiv zu bewerten und zu nutzen. Kurzes Abwenden, aber auch das kurze Verlassen einer verunreinigten Toilette, in der vielleicht ein "Zielpinkeln" veranstaltet wurde, hilft sich kurz zu sammeln und durchzuatmen. Dies gilt auch zum Beispiel vor der Reinigung von Erbrochenem.

\section{Gezielte Auszeiten nach belastenden Tätigkeiten}

Die Sensitivität für die Belastung nach Ekelsituationen ist in vielen Kita-Teams nicht ausgeprägt. Oft ist eine Fachkraft mit einer Ekel-Episode mit einem Kind recht lange beschäftigt und muss dann erleben, dass ihr, wenn gerade alles geschafft ist, gleich die nächsten Wickelkinder überantwortet werden, nach dem Motto: Du bist ja gerade dabei, hier ist noch einer!" Wickeln wie am Fließband ist für ein professionelles Ekelmanagement ungünstig. Oft wird die betroffene Fachkraft aber auch dringend zurückerwartet, weil in der Regel die Kollegin oder der Kollege allein mit den restlichen Kindern der Gruppe ist, weil der Morgenkreis ansteht oder der Übergang in den Garten. 
Hier ist es insgesamt wichtig im Team über kleine Minipausen nachzudenken, die nach ausgeprägten Ekel-Episoden eingeräumt werden könnten, wie kurz an die frische Luft gehen oder einen Kaffee/Tee trinken.

\section{Berufs)biographische Reflexionen - Psychohygiene durch Gespräche}

Für das Team ist es wichtig sich gegenseitig Ekelempfindungen zuzugestehen, und über das Etablieren regelmäßiger Gespräche einen offenen Umgang sicherzustellen. Gutknecht und Haug-Schnabel (2019) haben in ihrem Buch zur Begleitung des Kindes auf seinem Weg Ausscheidungsautonomie Windel adé! Reflexionsfragen entwickelt, die sich für einen familien- und berufsbiographischen Austausch eignen. Der Team-Dialog zum Thema Ekel dient auch der Psychohygiene der Mitarbeiter*innen:

- Wie wurde in Ihrer Familie mit dem Thema Ausscheidungsautonomie umgegangen? Welche Toilettenpraxis gab es?

- Wie wurde in Ihrer bisherigen Berufsbiografie mit dem Thema Ausscheidungsautonomie umgegangen?

- Wie leicht/schwer fällt es Ihnen, mit den Ausscheidungen der Kinder umzugehen?

- Gibt es Situationen, in denen Sie Ekel empfinden? Wie gehen Sie mit Ihren Ekelgefühlen um?

- Kennen Sie Situationen mit den Eltern der Kinder, in denen Sie Scham empfunden oder unwissentlich ausgelöst haben?

\section{Besonderheiten des Ekelmanagements auf der Heterogenitätsebene Behinderung}

Viele Kinder mit Behinderungen, Beeinträchtigungen oder Entwicklungsstörungen sind besonders gefährdet erst sehr spät die Ausscheidungsautonomie zu erreichen oder dauerhafte Probleme mit der Kontinenz zu entwickeln. Damit haben die Kinder ein großes Risiko, Ekel in ihrem Gegenüber auszulösen und Opfer von Übergriffen zu werden. Die Relevanz für ein Toilettentraining zur nachhaltigen Verbesserung der Lebensqualität, für nachhaltige Unabhängigkeit und Würde wird darum sehr hoch eingeschätzt.

Wichtig sind dabei auch die aktuellen Forschungsergebnisse zu den Zeitfenstern. So weiß man heute, dass Kinder, die bis zum Alter von 8 Jahren nicht toilettenfit sind, mit hoher Wahrscheinlichkeit keine Kontinenz erreichen (Cocchiola \& Redpath, 2017). Forschung hat zudem auch gezeigt, dass die Kinder ein deutlich höheres Maß an direkter Unterstützung und Anleitung benötigen und eine erheblich längere Lernzeit einzukalkulieren ist.

Die wissenschaftlichen Empfehlungen haben sich auf der Basis von Studien in Hinblick auf die Begleitung der Kinder auf ihrem Weg zur Ausscheidungsautonomie verändert (ebd. 2017). Beim Kind ohne Beeinträchtigung wird üblicherweise abgewartet, bis es von sich aus so genannte „Signale der Bereitschaft" zeigt wie beispielsweise das Interesse an der Toilette, an den 
Toiletten- aber auch Ausscheidungsvorgängen. Hier geht es darum, dass das Kind lernt die Signale aus seinem Körper wahrzunehmen und auf dieser Basis diese Signale auch nach außen zu melden.

Wird dieses Vorgehen kritiklos und ohne genaue Analyse auf Kinder mit Beeinträchtigungen übertragen, so kann dies dazu führen, dass keine Ausscheidungsautonomie aufgebaut wird. Cocchiola und Redpath (2017) schätzen Probleme beim Erwerb und bei der Aufrechterhaltung von Fähigkeiten im Bereich der Kontinenz-Entwicklung als erwartbar ein, wenn Kinder Probleme mit der neurophysiologischen Kontrolle und Koordination haben sowie Einschränkungen der kognitiven, sprachlichen und kommunikativen Fähigkeiten und der Emotionsregulation (vgl. auch Cocchiola et al., 2012, im Kontext Autismus, sowie Singh et al., 2016 im Kontext der Cerebralparese).

Singh, Masey, \& Morton (2016) empfehlen, mit einer verstärkten Unterstützung beim Erwerb der Ausscheidungsautonomie bald nach dem Alter von 3 Jahren einzusetzen. Gutknecht \& Haug-Schnabel empfehlen ein Alter von spätestens 5 Jahren, wenn nicht vorher bereits das Ergebnis vorliegt, dass das Kind aufgrund von organischen Störungen gar keine Signale aus seinem Körper wahrnehmen kann. Hier gibt es unterschiedliche Zeitangaben von Forschenden, aber prinzipiell wird ein früheres Eingreifen auf der Basis genauer Beobachtung und medizinischer Abklärung für erforderlich gehalten. Für die Bestimmung des Zeitpunktes sollten sich Eltern und pädagogische Fachkräfte auch mit dem Facharzt oder der Fachärztin für Pädiatrie abstimmen.

Weitere Forschungsergebnisse zeigen, dass ein Toilettentraining bei Kindern mit Beeinträchtigungen von den Fachkräften oft halbherzig oder unvollständig durchgeführt wird. Auch kommt es häufig zu einer unbeabsichtigten Verstärkung der Inkontinenz durch die Betreuungspersonen. Das Kind erfährt dann, dass es sich nicht lohnt, ausscheidungsautonom zu werden, weil es viel mehr Aufmerksamkeit und Zuwendung erhält, wenn es weiter einnässt und/oder einkotet (Cicero \& Pradt, 2002; Cooper, Heron, \& Heward, 2007).

\section{Fazit}

Die Bewältigung von Ekel auslösenden Pflege-Situationen ist ein Teil des regulären pädagogischen Alltags. Hilfreich sind daher für den Früh- und Elementarbereich die bisher überwiegend im Bereich der Pflegewissenschaften erarbeiteten Strategien zum Ekelmanagement (Pernlochner-Kügler, 2004; Ringel, 2017). Dies hat neben den zentralen Aspekten des Kinderschutzes auch den Hintergrund, dass der Versuch, Probleme mit der Emotion Ekel zu ignorieren, vorhandene Ekelgefühle beiseitezuschieben, falsch verstandene Idealvorstellungen einer Härte gegen sich selbst, zum Burnout führen können (Krey, 2017). Keine angehende pädagogische Fachkraft, kein/e Praktikant*in sollte unvorbereitet in Ekel-Situationen geraten, ohne Kenntnis über mögliche Bewältigungsstrategien. 
Eine Kita stellt jedoch ein anderes Setting als ein Krankenhaus dar. Daher ist es unabdingbar für den pädagogischen Bereich die eigenen, zum Teil bereits vorhandenen disziplinspezifischen Erkenntnisse zu identifizieren und weitere passgenaue Lösungsstrategien in den Blick zu nehmen und zu entwickeln (Gutknecht \& Haug-Schnabel, 2019). Dies impliziert auch, eine eigenständige Forschung zu den Themen Ekel und Scham im Setting einer Kindertageseinrichtung umzusetzen. Welche Ekel- und Schamepisoden treten im inklusiven Setting von Kindertageseinrichtungen genau auf? Wie wirken diese Ereignisse in der pädagogischen Arbeit mit den Kindern, in der Zusammenarbeit im Team, in der Zusammenarbeit mit Familien? Ekelepisoden können zu Responsivitäts-Verlusten führen, was als schwerwiegend in der pädagogischen Arbeit zu bewerten ist, da in der Responsivität der Fachpersonen der Hauptwirkfaktor in pädagogischen, aber auch therapeutischen Interventionen gesehen wird (Gutknecht, 2021). Wie lassen sich hier also Responsivitäts-Verluste vermeiden? Darüber muss eine inter- und transdisziplinäre Perspektive auf die Phänomene Ekel und Scham weiterentwickelt werden.

\section{Literatur}

Cicero, F. R. \& Pfadt, A. (2002). Investigation of a reinforcement-based toilet training procedure for children with autism. Research in Developmental Disabilities, 23(5), 319-331. https://doi.org/10.1016/S0891-4222(02)00136-1

Cocchiola, M. A. \& Redpath, C. (2017). Special Populations: Toilet Training Children with Disabilities. In Matson, J. (Ed.), Clinical Guide to Toilet Training Children: Autism, Child Psychopathology Series. Springer International Publishing. https://doi.org/10.1007/978-3-319-62725-0_13

Cocchiola, M. A., Martino, G. M., Dwyer, L. J. \& Demezzo, K. (2012). Toilet training children with autism and developmental delays: An effective program for school settings. Behavior Analysis in Practice, 5(2), 60-64. https://doi.org/10.1007/ BF03391824

Cooper, J. O., Heron, T. E. \& Heward, W. L. (2007). Applied behavior analysis (2nd ed.). Pearson Prentice Hall.

Ekman, P. (2010). Gefühle lesen: Wie Sie Emotionen erkennen und richtig interpretieren. Spektrum. https://doi.org/10.1007/978-3-662-53239-3

Gutknecht, D. (2021). Die Pikler Säuglings- und Kleinkindpädagogik im Fokus des wissenschaftlichen Diskurses. In Schmidt, T., Sauerbrey, U. \& Smidt, W: (Eds.), Frühpädagogische Handlungskonzepte - Eine kritische Bestandsaufnahme (pp. 129-148). Waxmann. S.

Gutknecht, D. (2020). Herausforderung Ekel in der Kita. Kindergarten Heute. Ausgabe, 11/12, 10-13.

Gutknecht, D. (2016). Einführung: Facetten der Inklusion. Themenheft Kleinstkinder in Krippe und Tagespflege: Vielfalt begrüßen. Inklusive Pädagogikfür die Jüngsten. 8-10. 
Gutknecht, D. (2015). Bildung in der Kinderkrippe. Wege zur Professionellen Responsivität. Kohlhammer.

Gutknecht, D. \& Haug-Schnabel, G. (2019). Windel adé. Kinder in Krippe und Kita achtsam begleiten. Herder.

Gutknecht, D., Stehmeier, S. \& Daldrop, K. (2018): Responsives Handling - Teil 1: Mehr als nur Windelwechseln. Kleinstkinder in Kita und Tagespflege. 6, 8-11.

Hadjittofi, M., Gleeson, K. \& Arber, A. (2020). The Experience of Disgust by Healthcare Professionals: A literature review. International Journal of Nursing Studies, 110(16):103720. https://doi.org/10.1016/j.ijnurstu.2020.103720

Häusser, L. F. (2012). Empathie und Spiegelneurone. Ein Blick auf die gegenwärtige neuropsychologische Empathieforschung. Praxis der Kinderpsychologie und Kinderpsychiatrie, 61(5), 322-335. https://doi.org/10.13109/prkk.2012.61.5.322

Holodynski, M. (2006). Emotionen - Entwicklung und Regulation. Springer.

Jettenberger, M. (2017). Ekel. Professioneller Umgang mit Ekelgefühlen in Gesundheitsfachberufen. Springer. https://doi.org/10.1007/978-3-662-54155-5_4

Kállo, È. (2015). Auf dem Weg zur Sozialisation: In Frieden mit mir - in Frieden mit anderen, In Tardos, A. \& Werner, A. (Eds.), Ich, Du und Wir. Frühes soziales Lernen in Familie und Krippe. 2. überarb. (pp. 85-101), Pikler-Gesellschaft.

Krey, H. (2015). Ekel ist okay: Ein Lern- und Lehrbuch zum Umgang mit Emotionen in Pflegeausbildung und Pflegealltag. 2. Aufl. Mabuse-Verlag. Kindle-Version.

Izard, C.E. (1999). Die Emotionen des Menschen: Eine Einführung in die Grundlagen der Emotionspsychologie. Beltz.

Mertens (2014). Handbuch psychoanalytischer Grundbegriffe. 4. Auflage, Kohlhammer. Kindle Ausgabe.

Özkan, I., Taylan, S., Adibelli, D.\& Yilmaz, F.T. (2021). Investigation of the Relationship between Nursing Students' Disgust Sensitivity and Caring Behaviours. Nurse Education in Practice, 54:103090. https://doi.org/10.1016/j.nepr.2021.103090

Pernlochner-Kügler, C. (2004). Körperscham und Ekel - wesentliche menschliche Gefühle. LIT Verlag.

Ringel, D. (2017). Ekel in der Pflege - eine, gewaltige‘ Emotion. 5. Aufl., Mabuse.

Rizzolatti, G. \& Sinigaglia, C. (2008). Empathie und Spiegelneurone. Die biologische Basis des Mitgefühls. Suhrkamp.

Rohrmann, T. \& Wanzeck-Sielert, C. (2018). Mädchen und Jungen in der Kita. Körper - Gender - Sexualität. Kohlhammer.

Singh, B., Masey, H. \& Morton, R. (2006). Levels of continence in children with cerebral palsy. Pediatric Nursing, 18(4), 23-26. https://doi.org/10.7748/ paed.18.4.23.s17

Waldenfels, B. (2007). Antwortregister. Suhrkamp. 